\title{
Letter to the \\ editor
}

\section{Diabetes insipidus is an unfavorable prognostic factor for response to glucocorticoids in patients with autoimmune hypophysitis}

\author{
Sabrina Chiloiro and Laura De Marinis \\ Pituitary Unit, Institute of Endocrinology, Catholic University of the Sacred Heart, Gemelli Foundation, Rome, Italy
}

Correspondence

should be addressed

to $S$ Chiloiro

Email

schiloiro@gmail.com

\section{Dear Editor,}

In a recent paper, Lupi and coworkers (1) described a retrospective series of 12 patients, affected by autoimmune hypophysitis, diagnosed at their Institution between 2008 and 2016. These patients were selected from 1500 patients for year evaluated within their Neuroendocrinology service. Lupi and coworkers diagnosed 4 cases of panhypophysitis (PH) and 8 cases of infundibuloneurohypophysitis (INH). In their series, all $\mathrm{PH}$ cases were affected by a multiple tropin defect, while in INH cases, the multiple tropin defect occurred in $25 \%$ of the cases, as well as a single tropin defect. Diabetes insipidus was identified in 50\% of the patients affected by PAH and in all patients affected by INH. The authors diagnosed GH and gonadotropin deficits in $50 \%$ of the cases, TSH deficit in $41 \%$ and ACTH deficit in $33 \%$. With regard to the pattern of autoimmunity and association with other autoimmune diseases, the authors detected immunofluorescence positivity for anti-pituitary autoantibodies in 50\% of the cases (3 PH and $3 \mathrm{INH}$ ) and association with other autoimmune diseases in none of the cases. Finally, the authors documented an improvement of pituitary function in around $50 \%$ of the patients. None of these patients was affected by diabetes insipidus.

Previously, we have also described a monocentric series of 21 patients affected by $\mathrm{PAH}$, observed between September 2011 and January 2015 at our tertiary care Pituitary Unit (2).

Unfortunately, Lupi and coworkers in their paper missed to compare the previous evidence proven by our work, which is the general rule when two papers represent a similar study (in this case, a monocentric Italian experience of patients affected by primary autoimmune hypophysitis). In fact, both papers $(1,2)$ present a similar study design with regard to the criteria applied for the definition of clinical diagnosis of $\mathrm{AH}$ as well as the criteria applied for excluding secondary causes of hypophysitis and different pituitary stalk lesions. Moreover, the two series were similarly investigated for the detection of coexisting autoimmune disease, for the definition of hypopituitarism (through the evaluation of the occurrence of single or multiple pituitary deficits) and for the patients' outcome.

In view of the similarities between these two papers and the studied populations, we think it is important for the Lupi and coworkers to compare the two cohorts of patients and the results of the studies in their paper and to discuss the similarities and the differences. This would contribute to improving knowledge on this very rare disease.

\section{References}

1 Lupi I, Cosottini M, Catureghi P, Manetti L, Urbani C, Cappellani D, Scattina I, Martino E, Marcocci C \& Bogazzi F. Diabetes insipidus is an unfavorable prognostic factor for response to glucocorticoids in patients with autoimmune hypophysitis. European Journal of Endocrinology 2017177 127-135 (https://doi.org/10.1530/EJE-17-0123)

2 Chiloiro S, Tartaglione T, Angelini F, Bianchi A, Arena V, Giampietro A, Mormando M, Sciandra M, Laino ME \& De Marinis L. An overview of diagnosis of primary autoimmune hypophysitis in a prospective single-center experience. Neuroendocrinology $2017 \mathbf{1 0 4}$ 280-290. (https://doi.org/10.1159/000446544)

Received 1 November 2017

Revised version received 15 November 2017

Accepted 15 November 2017 